\section{e0637 \\ A COMPARED STUDY OF FACILITATED PCI AND PRIMARY PCI ON ACUTE MYOCARDIAL INFARCTION VIA TRANSRADIAL ARTERY APPROACH}

doi:10.1136/hrt.2010.208967.637

Fu Xianghua, Liu Jun, Gu Xinshun, Wei Yongyun, Fan Weize, Wang Xuechao. The 2nd Hospital of Hebei Medical University. Shijiazhuang, Hebei, China

Objective To compare the effects and complications of facilitated PCI and primary PCI in AMI patients for probe the feasibility and safety of the facilitated PCI via transradial artery approach.

Methods Total of 94 first-time AMI patients were randomised to group A (facilitated PCI group, $\mathrm{n}=36$ ) and group $\mathrm{B}$ (primary PCI group, $\mathrm{n}=58$ ). In group $\mathrm{A}$, rt-PA $50 \mathrm{mg}$ thrombolytic treatment, then transferred to catherlab for CAG and PCI at once. In group B, the patients received primary $\mathrm{PCI}$ via transradial artery approach. In each group, recorded detail clinical information. Recorded LVEDP and left ventricular wall movement scores through LVG. All patients received ERNA and MIBI MPI in 1 week after PCI to evaluate the heart function and myocardial infarction area.

Results There was no significant differences about baseline data between two groups. The patients got reperfusion about $3 \mathrm{~h}$ earlier than group $\mathrm{B}$, and patients with TIMI 3 grade flow were more than group B before $(p<0.01)$ and after PCI $(p<0.05)$. No-reflow was fewer in group $A(p<0.05)$. And success rate of PCI was higher in the group $A(p<0.05)$. Myocardial perfusion was better in the group $A$, patients with TMP beyond 2 grade were more than group $B$ $(p<0.05)$. myocardial infarction area was smaller in the group $A$ $(p<0.01)$. The peak of CK and CK-MB were much lower in the group $A$ than group $B(p<0.01$, respectively). The ventricular wall movement Cortina scores and the incidence of ventricular aneurysms were lower in the group $\mathrm{A}$ than group $\mathrm{B}(\mathrm{P}<0.05$, respectively). LVEDP was lower in group A $(p<0.05)$, And the LVEF, left ventricular peak ejection rate, left peak filling rate in group $A$ were higher than group $B(p<0.01$, respectively), and left ventricular time to peak ejection rate, left ventricular time to peak filling rate were lower in the group $A(p<0.01$, respectively).

Conclusions The facilitated PCI via transradial artery approach has the benefit on the reduction of infarction area and protection of left ventricular function.

\section{e0638 CLINICAL EFFECTS AND SAFETY OF DELAYED PCI COMBINED WITH TIROFIBAN IN PATIENTS WITH ACUTE MYOCARDIAL INFARCTION}

doi:10.1136/hrt.2010.208967.638

Fu Xianghua, Gu Xinshun, Guo Xiaoping, Fan Weize, Jiang Yunfa, Hao Guozhen, Wu Weili, Li Shiqiang, Xue Ling, Jia Xinwei, Zhang Jing. The 2nd Hospital of Hebei Medical University, Shijiazhuang, Hebei, China

Objective To explore the clinical effects and safety of the delayed PCI combined with tirofiban compared with selective PCI in acute myocardial infarction (AMI) patients, so as to perform an earlier PCI for the AMI patients who missed the emergency time window and improve the prognosis

Methods A total of 80 patients with first AMI within $24 \mathrm{~h}$ to 7 days were randomised into two groups, the delayed PCI group (DPG, $\mathrm{n}=38$ ) and the control group (CG, $\mathrm{n}=42$ ). In DPG, tirofiban was administered $10 \mu \mathrm{g} / \mathrm{kg}$ within $3 \mathrm{~min}$ as a bolus followed by $0.15 \mu \mathrm{g} /$ $\mathrm{kg} / \mathrm{min}$, then transferred to the catherlab at once to perform CAG and PCI, while in CG, the patients received PCI to deal with infarctrelated artery 7-10 days after infarction. The clinical information, the haemorrhage events, MACE in hospital were collected. OCA and TMPG was used to analyse the coronary artery situation and the reperfusion of the IRA. The patients information in-hospital and on the $90^{\text {th }}$ day after PCI were compared.

Result There were no significant differences in clinical characteristics between the two groups. There was a greater percentage of TIMI 1 $(p<0.05)$ flow of IRA, but no significant difference in the percentage of TIMI 2 and TIMI 3 , in DPG than that in CG before PCI. The ratio of total occlusion lesion at the time of CAG, as well TIMI 0 flow of IRA after the guide wire first crossing, was significantly lower than that in CG (both $p<0.05$ ), while the percentage of TIMI 3 flow after stenting was higher $(p<0.05)$, the CTFC was fewer in DPG $(p<0.05)$, and the percentage of TMPG beyond 2 grade was higher in DPG $(p<0.05)$ than that in CG after stenting. There was no significant difference in LVEDVI, LVESVI, LVEF during in-hospital and in LVEF after 3 months of PCI ( $p>0.05$ ) between the two groups, while the LVEDVI, LVESVI were lower, LVEF was higher 3 months later in both groups. The platelet aggregation rate in DPG was lower after tirofiban administration. There was no significant difference in haemorrhage events and MACE between two groups.

Conclusions Delayed PCI combined with tirofiban may increase coronary blood flow, improve tissue-level perfusion and preserve the heart function, but did not obviously increase the haemorrhage events compared with selective PCI, which indicated that delayed PCI combined tirofiban may be an effective and safe way in the treatment of AMI.

\section{e0639 CLINICAL STUDY OF ENDEAVOUR STENT IN PATIENTS WITH CORONARY HEART DISEASE AND DIABETES}

doi:10.1136/hrt.2010.208967.639

Fu Xianghua, Liu Jinjun, Fan Weize, Jiang Yunfa, Wu Weili, Wang Yanbo. The 2nd Hospital of Hebei Medical University, Shijiazhuang, Hebei, China

Objective To evaluated the safety and efficacy of Endeavor Stent in patients with coronary heart disease (CHD) and diabetes performed percutaneous coronary intervention (PCI) via trans-radial artery approach for de novo lesions by comparing with Cypher stent.

Methods A total of 96 in-hospital patients with CHD and diabetes who performed PCI via trans-radial approach for de novo lesions were randomised into two groups: Endeavour group (EG, $n=52$ ), Cypher group (CG, $\mathrm{n}=44$ ). The minimal luminal diameter, diameter stenosis, incidence of restenosis and major adverse cardiac events of two groups instant and 6 months' follow-up after PCI were compared.

Results There are no significant differences between the two groups in baseline characteristics. There was no significant differences between the two groups in target-vessel distribution and ACC/AHA classification. $62.95 \%$ lesions treated were Type B2/C and $57.26 \%$ lesions were in left anterior descending artery. The average length of lesion, diameter of reference vessel, multivessel lesions, release pressure were no significant difference between the two group. Success rate of PCI was $100 \%$ in both of the two groups. All of the 96 patients were finished clinical follow up, among them, CAG were reperformed in 52 patients, 28 cases in EG and 24 cases in CG. The average follow-up period was no obvous difference between the two groups. There was no patient died in hospital. Each group had 1case happened AMI (for subacute thrombosis 3 days post-PCI in CG, and infarction of another vessel in EG. Three patients in EG, while four in CG occurred angina, two of which in each group were suffered from intra-stent restenosis. There was no significant difference between the two groups in minimal luminal diameter and diameter stenosis ( $p>0.05$ ) before, instantly after and 6 months after PCI, as well in early gain and late loss, restenosis rate and major adverse cardiac events ( $p$ all $>0.05$ ).

Conclusion Endeavour Stent was as safe and efficient as Cypher stent in patients with $\mathrm{CHD}$ and diabetes. 